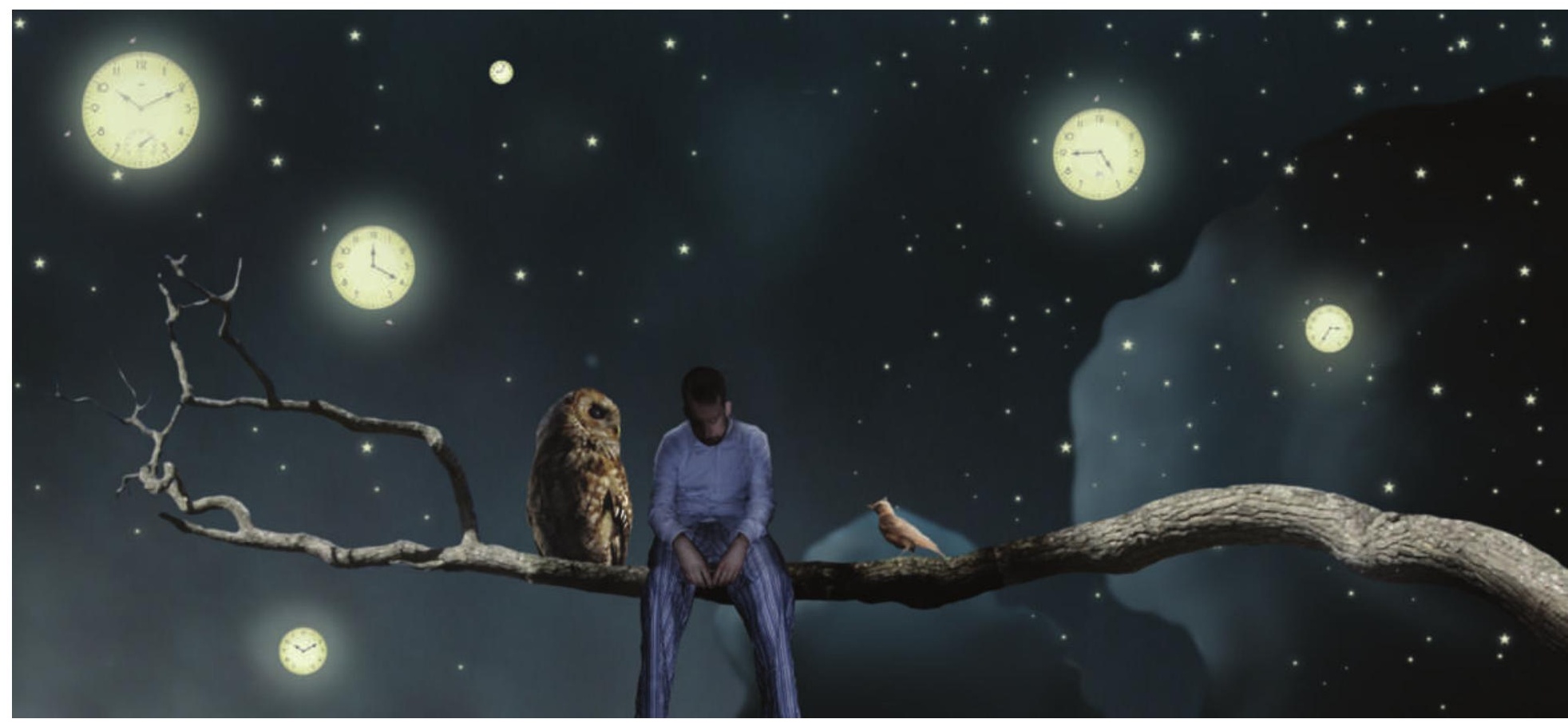

\title{
Of owls, larks and alarm clocks
}

\section{Could out-of-sync body clocks be contributing to human disease? Melissa Lee Phillips reports.}

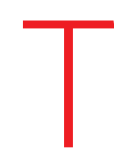
en years ago, researchers reported on three families with an extreme 'lark' problem. Larks are people who naturally wake up early in the morning, and are the opposite of 'owls', who wake up and go to sleep late. But many of the members of these families had particularly acute larkness, waking up on average around $4 \mathrm{a} . \mathrm{m}$. and falling asleep around 7.30 p.m. . The researchers later found that the families, who were diagnosed with familial advanced sleep-phase syndrome (FASPS), all carried mutations in a gene called PER2, which is involved in setting the human body clock ${ }^{2}$.

By some estimates, more than half of the population in industrialized societies may have circadian rhythms that are out of phase with the daily schedule they keep. Such people are said to have 'social jet lag' - a term coined by Till Roenneberg of Ludwig Maximilians University in Munich, Germany. Some of these are larks, some owls, and some have pretty standard human rhythms that are disrupted by shift work or travel. In modern societies, circadianrhythm disruptions can arise from simply spending too much time indoors, something that can make such workers decidedly "owlish", Roenneberg says. Even the one-hour time change made by many countries at this time of year can take some adjusting to.

If larks and owls are forced to follow normal schedules, they run into all kinds of problems with disabling insomnia and sleepiness. But disrupted rhythms could have graver consequences than that. In 2007, an expert working group at the World Health Organization's International Agency for Research on Cancer in Lyon, France, concluded that "shift-work that involves circadian disruption is probably carcinogenic to humans", after reviewing the existing evidence. Equally strong conclusions have been drawn from evidence that links circadian-rhythm problems to psychiatric disorders, metabolic syndrome and a range of other illnesses.

Researchers now are working to understand those links. Some suspect that health problems arise from a third kind of jet lag - one that arises when the circadian rhythms in different body tissues lose synchrony with each other. In 2006, the European Commission started funding EUCLOCK, a €16-million (US\$20million), five-year project involving some
34 researchers whose goal is to understand how the circadian clock synchronizes with cycles in the environment. In particular, the researchers are trying to work out what type of schedules are the healthiest fit for individuals' biological clocks. "People have been researching this for 50 years," says Anna Wirz-Justice at the Centre for Chronobiology in Basel, Switzerland, "but I think the methods are only now coming up to address this properly."

In nearly all organisms, patterns of biochemistry, physiology and behaviour oscillate with the daily cycles of light and dark, often with near-perfect timing. People forced to live in a 28-hour cycle still show fluctuations of almost exactly 24 hours in their core body temperature and levels of the hormones melatonin and cortisol ${ }^{4}$. In mammals, many of these cycles are directed by a 'master clock' in the brain's hypothalamus called the suprachiasmatic nucleus (SCN). The SCN receives information from the retina about light and coordinates rhythmic cycling of gene expression in the rest of the brain and body through neural signalling and hormones. Cycles of gene expression in 
the gastrointestinal tract, for example, ensure that digestive acids and enzymes are produced at the appropriate times.

In the past decade, researchers have identified some of the genes responsible for timekeeping, showing that expression of CLOCK, $B M A L 1, P E R$ and CRY rises and falls over 24 hours in the SCN and elsewhere, and that mice with mutated versions of these genes abandon the 24-hour cycle for one that is shorter, longer or has no pattern at all. Such animals were "the key development that brought the field to its present exciting position", WirzJustice says, because it suggested that these were 'master genes' directing the clock and the physiological processes that follow it.

\section{Typecasts}

But for some individuals, the cycles of gene expression and behaviour do not adhere well to the cycles of night and day. The tendency to be a lark, an owl, or somewhere in between is referred to as individual's 'chronotype', and although it may shift over the course of a person's lifetime - adolescents and young adults tend to be more owl-like than either children or older adults - it doesn't usually change in comparison with peers and it is thought to be determined largely by genes. FASPS was the first human circadian disorder linked to a mutation in a specific clock gene. Not all such genes have been easy to find: researchers have found no simple mutation that accounts for people with the owlish delayed sleep-phase syndrome (DSPS), who can have trouble falling asleep before 6 a.m. and waking up before 2 p.m..

\section{People with DSPS and} FASPS often also have depres$\operatorname{sion}^{5,6}$, and this and other psychiatric conditions, such as bipolar disorder and schizophrenia, are commonly associated with abnormalities in circadian rhythms. "The vast

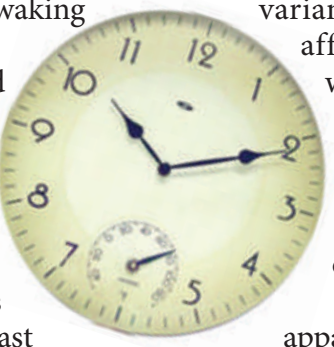

Circadian rhythms are known to affect the most basic of metabolic pathways, including protein synthesis, glycolysis and fatty-acid metabolism. And many patients who have circadian-rhythm disorders caused by shift work also develop gastrointestinal and metabolic disorders such as glucose intolerance, diabetes and high blood pressure, says Theodore Bushnell, a neurologist at the University of Washington Medicine Sleep Disorders Center in Seattle. "It seems like there's nobody who just has a shift-work issue."

The conclusion that shift work can be carcinogenic has grown largely from epidemiological work. A nationwide study in Denmark, for example, found that women who work mainly at night for at least six months are 1.5 times more likely to develop breast cancer than those who work regular hours ${ }^{8}$. Researchers sug gest that the raised cancer risk could be because these people's cells start to divide at the wrong time and run amok, an idea supported by some cell-culture studies.

So far, these links haven't provided the details that biologists would like. Much like diet or stress, circadian rhythms affect so many cellular and physiological functions that it is extremely difficult to pinpoint a mechanism by which a given alteration to these rhythms could contribute to a particular disease. Much of the current work involves genetic association studies, in which scientists look for gene variants that pop up more often in people affected with a disorder than in those without it. Some such studies for psychiatric diseases, for example, have pulled out variants in clock genes. But researchers have also been making progress by dissecting the machinery of the various human body clocks.

Since the late 1990s, it has become apparent that the body has 'peripheral cirmajority of people with major depression have sleep abnormalities and interestingly it can be that they sleep too much or they have insomnia and can't sleep," says Colleen McClung of the University of Texas Southwestern Medical Center in Dallas. This connection raises a cause and effect question: are the circadianrhythm disorders causing the depression or the other way around? In 2007, McClung and her colleagues found some support for the former idea when they studied mice that lack a working Clock gene and, they observed, exhibit symptoms of mania and hyperactivity that can be reversed by the mood stabilizer lithium 7 . "Every way we test them, they look like bipolar patients in the manic state," McClung says. cells followed a clock of 24.5 hours, whereas the corneal cells ran at about 21.5 hours ${ }^{9}$. "We used to think the clock was just in the brain - it was a neural process and the body just passively followed," Takahashi says. "But that's clearly not the case. You have to view your

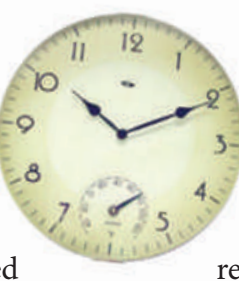

body as a whole collection of different clocks."

Like Greenwich Mean Time for the body, the SCN serves as a reference point for the peripheral clocks, and they usually run in sync. But sometimes - after stepping off a long-distance flight, for example - the synchrony breaks down. When simulating jet lag in rats, researchers at the University of Tokyo found that the SCN resets to local time in around one day based on light signals, but peripheral oscillators can take more than a week to adjust ${ }^{10}$. "So, during jet lag, your body is literally completely out of sync," Takahashi says. "Each organ shifts at a different rate."

Could it be that loss of synchrony between the body's tissues underlies some of the health problems seen in circadianrhythm disorders and shift workers? To test this, researchers have used genetic tools to create an artificial mismatch between the central clock and a peripheral one. In one study $^{11}$, researchers knocked out the gene Bmal1 in the liver of mice, effectively disabling the clock in that organ. When the animals developed low blood sugar for parts of the day, the researchers knew that the brain clock was not sufficient to maintain glucose levels - a working peripheral clock was needed too.

The SCN is synchronized during the daytime by light, whereas liver metabolism is also synchronized by food intake, Roenneberg says. So perhaps shift-workers have gastrointestinal problems because their liver and intestinal tracts are gearing up for a meal at the wrong time. "You can easily imagine that this is not exactly optimal for the system," he says.

\section{Out of time}

A mismatch between central and peripheral clocks has been linked to other health conditions too. Ongoing work by Christian Cajochen at the Centre for Chronobiology in Basel, suggests that the peripheral clocks of women with depression are not as well linked to sleep-wake cycles as they are in those without the condition. "Women with depression have a greater degree of variability in the timing of different physiological and endocrine rhythms," Wirz-Justice says.

Some researchers in the EUCLOCK consortium are planning to work out how the

SCN is able to keep peripheral clocks and

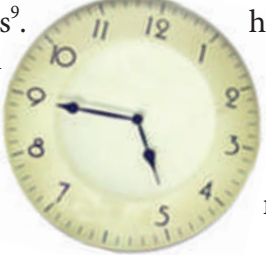

human physiology in time. In some experiments, mice will be put onto 'shift-work' schedules in which they are forced to 'work' and eat during the day rather than at night. The researchers will then examine how the 


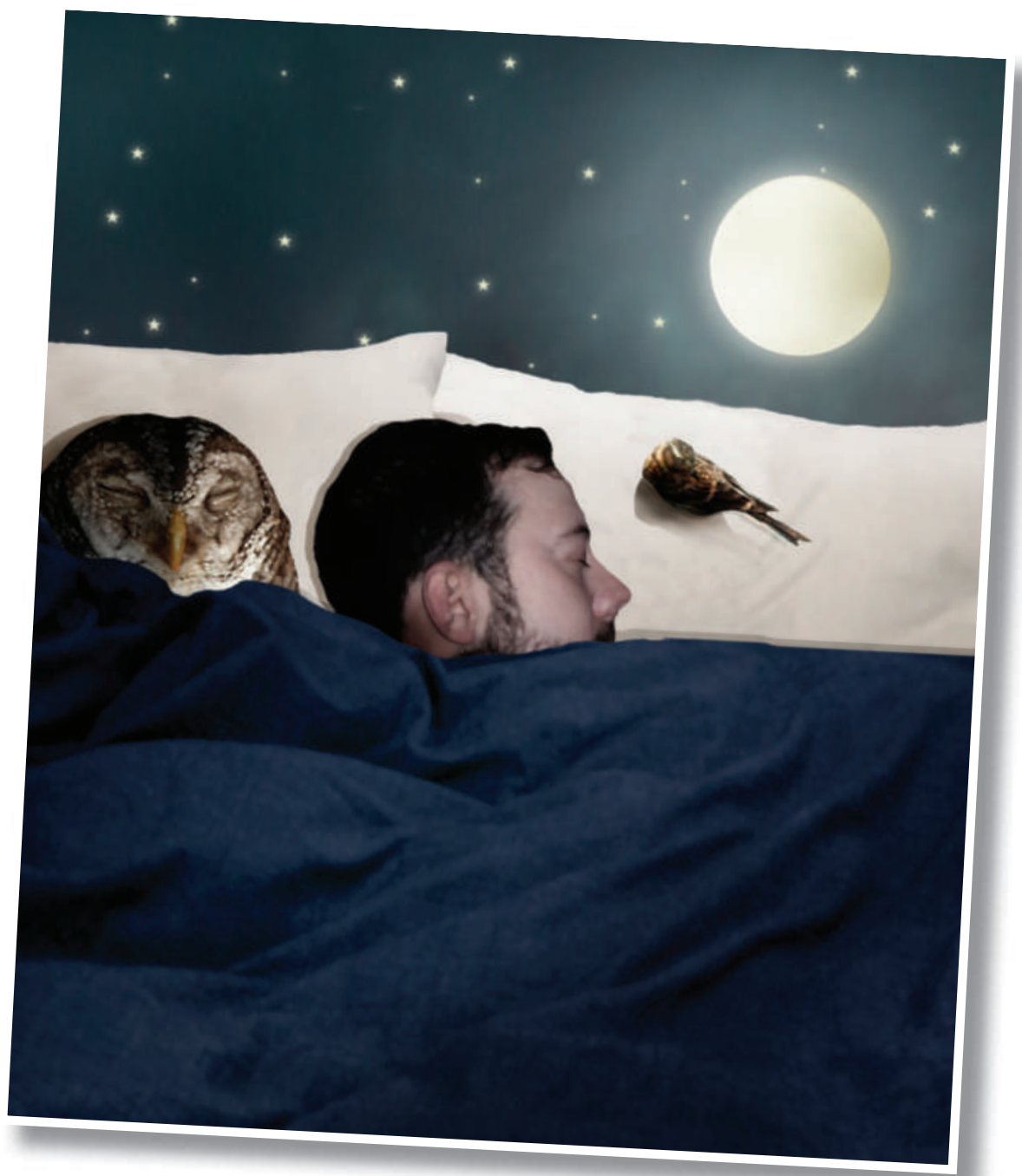

schedule affects behaviour and the synchronization of various local body clocks.

In fact, peripheral oscillators have already proved to be a useful research tool. Last February, researchers from Steven Brown's lab at the University of Zurich and Achim Kramer's group at Charité Hospital in Berlin identified 11 larks and 17 owls based on a 'morningnesseveningness' questionnaire and then measured their molecular rhythms from the expression of Bmall in their skin cells ${ }^{12}$. The team wanted to find out why larks and owls naturally adopt the schedules that they do. For some people, the researchers found what they expected: cells from larks showed shorter periods than those from owls. But they also found that about half of the

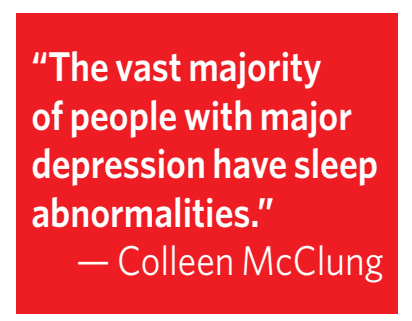

length but also from differences in how easily a person's rhythms can be synchronized to the night-day cycle - and that some larks and owls have clocks that are not reset normally each day. So perhaps these people's peripheral clocks stray from the central one easily.

\section{Therapy options}

With few firm mechanisms to go on, the question now is how to go about 'treating' circadian-rhythm disruptions. Intense-light therapy has been used to shift undesirable sleep schedules back to a more normal pattern. And WirzJustice says that researchers are working on other behavioural or pharmaceutical ways to alter circadian rhythms. Ramelteon, for instance, is a larks and owls actually had 'normal' circadian period lengths.

The researchers found that in this group, the owls had skin clocks that were more difficult to reset than those of people with more typical schedules, and that the larks had clocks that were easier to reset. This suggests that individual differences in chronotype result not just from innate differences in circadian period drug used to reduce the effects of insomnia by mimicking the action of melatonin - a hormone that tells the body it is sleep time. But no one knows yet whether such interventions will also prevent or reduce some of the other health risks associated with circadian-rhythm disruptions. Roenneberg is not convinced that it would be easy to solve even conventional jet lag with drugs. "The trouble is that we are still much too naive to have pharmacological success without messing something else up in the system," he says.

That means it could be up to individuals to consider their chronotype before choosing their schedule, or making other decisions that might affect their health. EUCLOCK researchers are developing ways to measure clock-gene expression from cheek swabs or other tissue that could provide a quick laboratory read-out of a person's chronotype. (Roenneberg says that so far these results agree with self-reported chronotype based on questionnaires.) Chronotype might be taken into account when administering drugs or medical tests: performing a blood test at $8 \mathrm{a} . \mathrm{m}$. can yield completely different results on a morning person, who has been up for hours, than it will on an evening one. "You can get very different clinical blood values based on their chronotypes without anything being meaningfully different, because in one person, the system is up, and in the other, it's still down," says Roenneberg.

Working with EUCLOCK and another consortium called CLOCKWORK, Roenneberg and his colleagues are developing a computer model that works out a person's ideal schedule on the basis of his or her chronotype and sleep needs. The group is starting to test the model's predictions by asking people of each chronotype to try out a certain schedule in their daily lives, and measuring its effects in a range of physiological, behavioural and cognitive tests. They will then use their findings to refine the model. By repeating this process several times, Roenneberg says, "we are confident that we will finally put some sense into how to do shiftwork properly - that is, with the fewest side effects on health".

Being a lark or owl should not, in itself, be a problem, Roenneberg adds. "Chronotype per se should have no health effects whatsoever. Health effects come from having to live against one's own clock."

Melissa Lee Phillips is a freelance writer based in Seattle, Washington.

1. Jones, C. R. et al. Nature Med. 5, 1062-1065 (1999).

2. Toh, K. L. et al. Science 291, 1040-1043 (2001).

3. International Agency for Research on Cancer Lancet Oncol. 8, 1065-1066 (2007).

4. Czeisler, C. A. et al. Science 284, 2177-2181 (1999)

5. Shirayama, M. et al. Sleep Med. 4, 427-433 (2003).

6. Xu, Y. et al. Nature 434, 640-644 (2005).

7. Roybal, K. et al. Proc. Natl Acad. Sci. USA 104, 6406-6411 (2007).

8. Hansen, J. Epidemiology 12, 74-77 (2001).

9. Yoo, S.-H. et al. Proc. Natl Acad. Sci. USA 101, 5339-5346 (2004).

10. Yamazaki, S. et al. Science 288, 682-685 (2000).

11. Lamia, K. A., Storch, K. F. \& Weitz, C. J. Proc. Natl Acad. Sci. USA 105, 15172-15177 (2008).

12. Brown, S. A. et al. Proc. Natl Acad. Sci. USA 105, 1602-1607 (2008) 